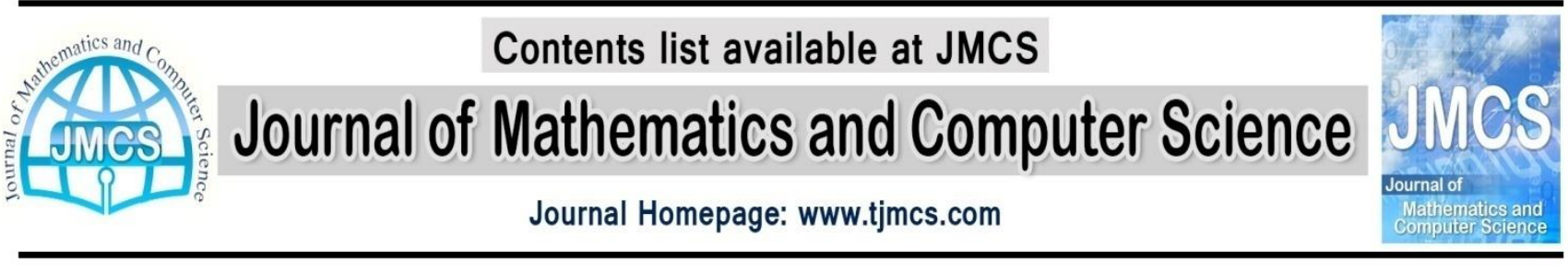

\title{
Stabilization of Dynamic Systems by Localization of Eigenvalues in a Specified Interval
}

\author{
H. Ahsani Tehrani \\ Department of Mathematics, Shahrood University of Technology, Shahrood, Iran. \\ hahsani@shahroodut.ac.ir
}

Article history:

Received March 2013

Accepted Apri 2013

Available online April 2013

\section{Abstract}

This paper is concerned with the problem of designing linear time-invariant control systems with closed-loop eigenvalues in a prescribed region of stability. First, we obtain a state feedback matrix which assigns all the eigenvalues to zero, and then by elementary similarity operations we find a state feedback which assigns the eigenvalues in the interval shown in figure 1.

This new algorithm can also be used for the placement of closed-loop eigenvalues in a specified interval in z-plane and can be employed for large-scale linear time-invariant control systems. Some illustrative examples are presented to show the advantages of this new technique.

Keywords: linear time-invariant systems; State feedback matrix; Localization of eigenvalues; interval; Large-scale systems

\section{Introduction}

In many applications, mere stability of the controlled object is not enough, and it is required that the poles of the closed-loop system should lie in a certain restricted region of stability. Several design methods have been reported which utilize the LQ technique to achieve the desired pole allocation Amin [1] derived an improved result in which the optimality of the closed-loop system is assured. Furuta and Kim [7] obtained a method for assigning the closed loop poles in a specified disk based on gain and phase margins which is named -stability margin. They considered the case, when the perturbations are unknown gains as a diagonal form. Yuan and Achenie and Jiang [14] addressed the problem of linear quadratic regulator (LQR) synthesis with regional closed-loop pole constraints. Determining the objective value range for a class of interval convex 
optimization problems is introduced in [9]. Figueroa and Romagnoli [6] presented a method for designing controllers which attempt to place the roots of a characteristic polynomial of an uncertain system inside some prescribed regions. The analysis is based on the transfer function of a characteristic polynomial. Chou [4] described another pole assignment method with a spectral radius and proposed a pulse transfer function. The procedure is simple, but it is used only for checking the positions of closed loop poles, not for designing the controller. Benner and Castillo and Quintana-Orti [3] presented the method for partial stabilization of large-scale discrete-time linear control systems. Grammont and Largillier [8] employed an approach to localize matrix eigenvalues in the sense that they build a sufficiently small neighborhood for each eigenvalue (or for a cluster). Arjmandzadeh and Effati and Zamirian [2] proposed an interval support vector regression (ISVR) problem which the training samples are interval values.

A well-known desired region for continuous systems is left side of complex plan. In the simplest case, the real parts of all closed-loop eigenvalues are required to be into interval $(a, b)$ where $a$ and $b$ are real numbers and $b<a<0$. Generally, the more practically important region the closed-loop poles is the interval shown in figure 1. In this paper, the aim is to present a method for localization of eigenvalues in specified region of complex plane by state feedback control for large-scale continuous-time linear dynamic systems.

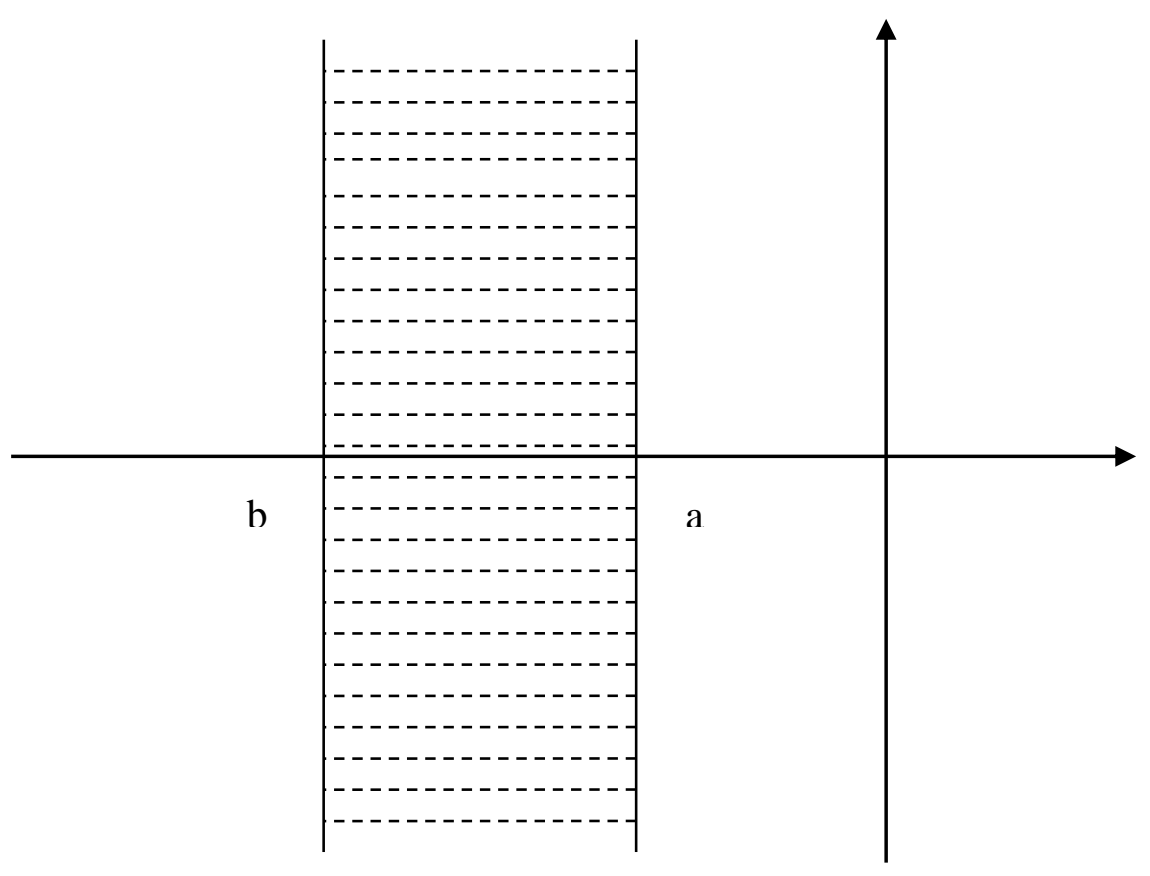

Figure 1. Specified interval 


\section{Problem Statement}

The problem of localization of eigenvalues in a small specified region has been the subject of many investigators in the last decade [3, 8].

Consider a controllable linear time-invariant dynamic system defined by the state equation

$$
\dot{x}(t)=A x(t)+B u(t)
$$

where $x(t) \in \mathfrak{R}^{n}, u(t) \in \mathfrak{R}^{m}$ and the matrices $A$ and $B$ are real constant matrices of dimensions $n \times n$ and $n \times m$ respectively, with $\operatorname{rank}(B)=m$. The aim of eigenvalue assignment in a specified region is to design a state feedback controller, $K$ producing a closed-loop system with a satisfactory response by shifting controllable poles from undesirable to desirable locations. Karbassi and Bell [10, 11], have introduced an algorithm for obtaining an explicit parametric controller matrix $K$ by performing similarity operations on the controllable pair $(B, A)$. In fact, $K$ is chosen such that the closed-loop system eigenvalues

$$
\Gamma=A+B K
$$

lie in the self-conjugate eigenvalue spectrum $\Lambda=\left\{\lambda_{1}, \lambda_{2}, \ldots, \lambda_{n}\right\}$. Recently, Karbassi and Tehrani [12] extended the previous results as to obtain an explicit formula involving nonlinear parameters in the control law. The stabilization problem consists in finding a feedback matrix $K \in \mathfrak{R}^{m \times n}$ such that the input $u(t)=K x(t)$, yields a stable closed loop system

$$
x(t)=x(0) \exp (\Gamma t)
$$

In case the spectrum (or set of eigenvalues) of the closed-loop matrix, denoted by $\Lambda(\Gamma)$, is contained in the left side of complex plan we say that $\Gamma$ is (Schur) stable or convergent. The stabilization problem arises in control problem such as, the computation of an initial approximate solution in Newton's method for solving discrete-time algebraic Riccati equations, simple synthesis methods to design controllers. Large-scale problems occur whenever the linear system results from some sort of a partial differential equation or from delay systems. There, the number of states is often a couple of thousands.

The stabilization problem can in principal be solved as a eigenvalue assignment problem. eigenvalue assignment methods compute a feedback matrix such that the closed-loop matrix of system (2) has a prespecified spectrum. In this paper, we present an efficient approach for localization of eigenvalues in specified region for large-scale linear continuous-time systems. Our assignment procedure is composed of two stages. We first obtain a primary state feedback matrix $F_{p}$ which assigns all the eigenvalues of closedloop system to zero, then produce a state feedback matrix $K$ which assigns all the closedloop eigenvalues in specified interval. 


\section{Synthesis}

Consider the state transformation

$$
x(t)=T \tilde{x}(t)
$$

where $T$ can be obtained by elementary similarity operations as described in [10]. In this way, $\widetilde{A}=T^{-1} A T$ and $\widetilde{B}=T^{-1} B$ are in a compact canonical form known as vector companion form:

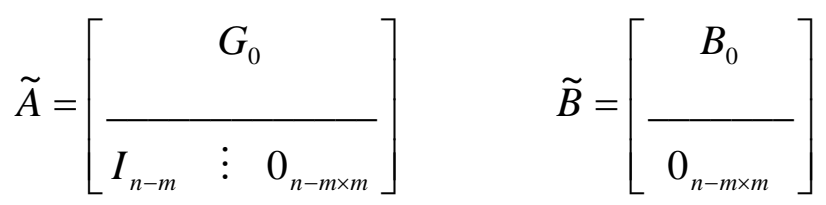

Here $G_{0}$ is an $m \times n$ matrix and $B_{0}$ is an $m \times m$ upper triangular matrix. Note that if the Kronecker invariants of the pair $(B, A)$ are regular, then $\tilde{A}$ and $\widetilde{B}$ are always in the above form [10]. In the case of irregular Kronecker invariants, some rows of $I_{n-m}$ in $\tilde{A}$ are displaced [11]. It may also be concluded that if the vector companion form of $\tilde{A}$ obtained from similarity operations has the above structure, then the Kronecker invariants associated with the pair $(B, A)$ are regular [10].

The state feedback matrix which assigns all the eigenvalues to zero, for the transformed $\operatorname{pair}(\tilde{B}, \tilde{A})$, is then chosen as

$$
u=-B_{0}^{-1} G_{0} \tilde{x}=\tilde{F} \tilde{x}
$$

Which results in the primary state feedback matrix for the pair $(B, A)$ defined as

$$
F_{p}=\tilde{F} T^{-1}
$$

The transformed closed-loop matrix $\tilde{\Gamma}_{0}=\tilde{A}+\tilde{B} \tilde{F}$ assumes a compact Jordan form with zero eigenvalues

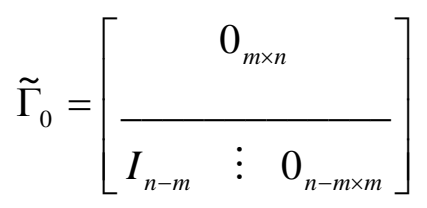

Theorem 1: Let $D$ be a block diagonal matrix in the form

$$
D=\left[\begin{array}{cccc}
D_{1} & 0 & \cdots & 0 \\
0 & D_{2} & \cdots & 0 \\
\vdots & \vdots & \ddots & \vdots \\
0 & 0 & \cdots & D_{k}
\end{array}\right]
$$

where each $D_{j},(j=1,2, \ldots, k)$ is either of the form 


$$
D_{j}=\left[\begin{array}{cc}
\alpha_{j} & \beta_{j} \\
-\beta_{j} & \alpha_{j}
\end{array}\right]
$$

(To designate the complex conjugate eigenvalues $\alpha_{j}+i \beta_{j}$ ) or in case of real eigenvalues

$$
D_{j}=\left[d_{j}\right]
$$

If such block diagonal matrix $D$ with self-conjugate eigenvalue spectrum is added to the transformed closed-loop matrix, $\widetilde{\Gamma}_{0}$ then the eigenvalues of the resulting matrix is the eigenvalues in the spectrum.

Proof: The primary compact Jordan form in the case of regular Kronecker invariants is in the form

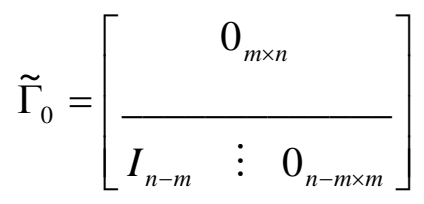

The sum of $\tilde{\Gamma}_{0}$ with $D$ has the form:

$$
\begin{gathered}
\tilde{H}=\tilde{\Gamma}_{0}+D \\
=\left[\begin{array}{ccc}
0_{m \times n} & \\
\hline I_{n-m} & \vdots & 0_{n-m \times m}
\end{array}\right]+\left[\begin{array}{cccc}
D_{1} & \cdots & 0 \\
\vdots & \ddots & \vdots \\
0 & \cdots & D_{k}
\end{array}\right] \\
=\left[\begin{array}{ccccccc}
D_{1} & 0 & \cdots & 0 & 0 & \cdots & 0 \\
0 & D_{2} & \cdots & 0 & 0 & \cdots & 0 \\
\vdots & & \ddots & \vdots & \vdots & \vdots & \vdots \\
0 & 0 & \cdots & D_{l} & 0 & \cdots & 0 \\
I_{1} & 0 & \cdots & 0 & D_{l+1} & \cdots & 0 \\
\vdots & \ddots & \cdots & 0 & 0 & \ddots & \vdots \\
0 & \cdots & I_{r} & 0 & 0 & \cdots & D_{k}
\end{array}\right]
\end{gathered}
$$

where $I_{s}, s=1,2, \ldots, r$ is the unit matrix of size 2 in case $n-m$ is even. In case $n-m$ is odd only one $I_{s}$ takes the form of a unit matrix of size one. 
By expanding $\operatorname{det}(\tilde{H}-\lambda I)$ along the first row it is obvious that the eigenvalues of $\tilde{H}$ are the same as the eigenvalues of $D$. For the case of irregular Kronecker invariants [11] only some of the unit columns of $I_{n-m}$ are displaced, since the unit elements are always below the main diagonal, the proof applies in the same manner.

\section{Corollary}

Then $\tilde{H}_{\lambda}$ can be obtained from $\tilde{H}$ by performing elementary similarity operations

$$
\operatorname{Column}(j)-\lambda_{j} \operatorname{Column}(i)
$$

followed by

$$
\operatorname{Row}(i)+\lambda_{j} \operatorname{Row}(j)
$$

for $\mathrm{j}=\mathrm{n}, \mathrm{n}-1, \ldots, \mathrm{m}, \mathrm{i}=\mathrm{j}-\mathrm{m}$.

Hence, the matrix $\tilde{H}_{\lambda}$ thus obtained will be in primary vector companion form such that:

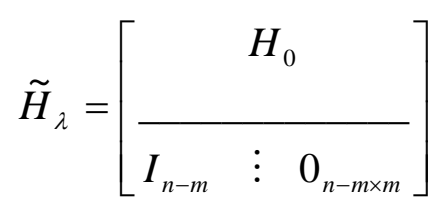

where $H_{0}$ is an $m \times n$ matrix .

Because of similarity operation, the eigenvalues of the matrix $\tilde{H}_{\lambda}$ are the same as the eigenvalues of $\tilde{H}$ and that of $D$. Now the feedback matrix of the pair $(\tilde{A}, \tilde{B})$ is defined by:

$$
\tilde{K}=\tilde{F}+B_{0}^{-1} H_{0}=B_{0}^{-1}\left(-G_{0}+H_{0}\right)
$$

Theorem 2: The state feedback matrix $\tilde{K}$ assigns the eigenvalues of closed-loop matrix $\tilde{\Gamma}=\tilde{A}+\tilde{B} \tilde{K}$ in the interval shown in figure 1 if we suppose $\alpha_{j}, \beta_{j}, d_{j}$ be in the form:

$$
\begin{aligned}
& \alpha_{j}=-|b-a| * \operatorname{random}(0,1)+a \\
& \beta_{j}=k * \operatorname{random}(0,1) \quad k \in \mathrm{Z}
\end{aligned}
$$

and for assigning real valued eigenvalues in the interval shown in Figure 1, we choose

$$
d_{j}=-|b-a| * \operatorname{random}(0,1)+a
$$

Proof: The eigenvalues of matrix $D$ defined above fall in the specified interval.

Let

$$
\tilde{\Gamma}=\tilde{A}+\tilde{B} \tilde{K}=\left[\begin{array}{lll} 
& G_{0} & \\
I_{n-m} & & 0_{n-m, m}
\end{array}\right]+\left[\begin{array}{c}
B_{0} \\
0_{n-m, m}
\end{array}\right]\left[B_{0}^{-1}\left(-G_{0}+H_{0}\right)\right]
$$




$$
\tilde{\Gamma}=\left[\begin{array}{lll} 
& G_{0}-B_{0} B_{0}^{-1} G_{0}+B_{0} B_{0}^{-1} H_{0} & \\
I_{n-m} & 0_{n-m, m}
\end{array}\right]=\left[\begin{array}{lll} 
& H_{0} & \\
I_{n-m} & & 0_{n-m, m}
\end{array}\right]
$$

Clearly $\widetilde{\Gamma}=\tilde{H}_{\lambda}$, since $\tilde{H}_{\lambda}$ is similar to the matrix $\tilde{H}$ and the eigenvalues of matrix $\tilde{H}$ are the same as that of matrix $D$ and elementary similarity operations do not change the eigenvalues, then the eigenvalues of closed-loop matrix $\widetilde{\Gamma}=\tilde{A}+\widetilde{B} \widetilde{K}$ fall in the specified interval.

Remark: Since $\tilde{K}$ assigns the eigenvalues of the closed-loop matrix $\tilde{\Gamma}=\tilde{A}+\tilde{B} \tilde{K}$ in the specified interval it is obvious that the state feedback controller matrix, $K=\tilde{K} T^{-1}=B_{0}^{-1}\left(-G_{0}+H_{0}\right) T^{-1}$ also assigns the eigenvalues of the closed-loop matrix $\Gamma=A+B K$ in the specified interval too.

Note for assign the eigenvalues of the closed-loop matrix in spectrum $\Lambda=\left\{\lambda_{1}, \lambda_{2}, \ldots, \lambda_{n}\right\}$ we suppose

$$
D_{j}=\lambda_{j} \quad j=1,2, \ldots, n
$$

\section{An algorithm for assignment of eigenvalues in the interval shown in figure 1}

In this section we first give an algorithm for finding a state feedback matrix which assigns zero eigenvalues to the closed-loop system. Then we determine a gain matrix which assigns the closed-loop eigenvalues in specified interval.

Input: The controllable pair $(A, B)$, the primary state feedback $F_{p}, B_{0}^{-1}$ and $T^{-1}$ which are calculated by the algorithm proposed by Karbassi and Bell [10,11], the $\theta$ angle and distance angle apex of the origin of the complex plane $(a)$.

Step 1. Construct the block diagonal matrix $D$ in the form (9), in which for assigning complex valued eigenvalues in the interval shown in Figure 1 we choose

$$
\begin{aligned}
& \alpha_{j}=-|b-a| * \operatorname{random}(0,1)+a \\
& \beta_{j}=k * \operatorname{random}(0,1) \quad k \in \mathrm{Z}
\end{aligned}
$$

and for assigning real valued eigenvalues in the interval shown in Figure 1, we choose

$$
d_{j}=-|b-a| * \operatorname{random}(0,1)+a
$$

Step 2. Set

$$
\tilde{H}=\widetilde{\Gamma}_{0}+D
$$

Step 3. Transform $\tilde{H}$ to primary vector companion form $\tilde{H}_{\lambda}$ as in (18) using elementary similarity operations as specified in corollary of theorem 1 . 
step 4. Now compute $K=F_{p}+B_{0}^{-1} H_{0} T^{-1}$ the required state feedback matrix.

\section{Illustrative Examples}

Consider a large discrete-time system given by

$$
\dot{x}(t)=A x(t)+B u(t)
$$

Where $A$ and $B$ are randomly generated with $n=10$ and $m=6$.

$$
A=\left[\begin{array}{llllllllll}
2 & 5 & 6 & 0 & 8 & 7 & 4 & 1 & 0 & 8 \\
7 & 6 & 0 & 1 & 9 & 6 & 9 & 5 & 8 & 9 \\
6 & 6 & 0 & 3 & 1 & 8 & 2 & 4 & 0 & 3 \\
2 & 6 & 0 & 2 & 8 & 2 & 8 & 6 & 5 & 4 \\
6 & 8 & 2 & 7 & 9 & 4 & 6 & 6 & 1 & 4 \\
6 & 0 & 5 & 3 & 1 & 2 & 8 & 9 & 7 & 1 \\
6 & 3 & 4 & 6 & 1 & 0 & 4 & 1 & 2 & 1 \\
1 & 7 & 7 & 9 & 7 & 0 & 9 & 1 & 9 & 5 \\
6 & 3 & 5 & 5 & 7 & 8 & 3 & 5 & 1 & 7 \\
1 & 9 & 5 & 9 & 6 & 3 & 5 & 9 & 5 & 3
\end{array}\right] \quad B=\left[\begin{array}{llllll}
3 & 1 & 2 & 6 & 7 & 1 \\
2 & 0 & 3 & 8 & 3 & 1 \\
8 & 2 & 0 & 5 & 4 & 4 \\
6 & 1 & 8 & 9 & 4 & 4 \\
2 & 0 & 8 & 7 & 6 & 0 \\
9 & 8 & 2 & 1 & 1 & 9 \\
6 & 1 & 5 & 8 & 0 & 1 \\
2 & 0 & 1 & 1 & 7 & 3 \\
6 & 7 & 5 & 6 & 5 & 3 \\
6 & 5 & 3 & 6 & 2 & 1
\end{array}\right]
$$

The open loop eigenvalues are

$\{2.0345 \pm 1.8326 i,-3.3771 \pm 5.3252 i,-6.4060 \pm 5.6907 i, 0.6634,6.8249,-8.2872,46.2962\}$ which are widely spread in the complex plane. In order to locate them in small discs inside the unit circle, we employ the above algorithm step by step. First, the primary state feedback matrix which locates all the eigenvalues of the closed-loop system to the origin of the complex plane is found to be:

$F_{p}=\left[\begin{array}{cccccccccc}-50.9331 & -19.5738 & 18.5282 & -14.5585 & 0.2820 & -26.0893 & -10.8780 & -38.9380 & 1.7136 & 2.8572 \\ 24.7556 & 10.4052 & -9.1386 & 7.6143 & 0.3770 & 11.6431 & 6.9068 & 19.2376 & 0.9310 & -1.4368 \\ -45.5535 & -17.7300 & 16.3422 & -13.8318 & -1.3198 & -22.1965 & -11.6130 & -35.6428 & -0.6945 & 2.0236 \\ 54.1703 & 20.5013 & -19.2125 & 16.1558 & 0.3089 & 26.7532 & 12.6628 & 41.4999 & -0.2578 & -2.8454 \\ 3.1621 & 1.1667 & -2.5934 & -1.1326 & -0.4128 & 1.9142 & 0.5168 & 4.5242 & -0.9917 & -1.1463 \\ 2.9122 & 0.2399 & -1.2857 & 2.8436 & -1.3767 & 1.5498 & -2.1781 & -1.0741 & -1.7146 & -0.2804\end{array}\right]$

It is desired to locate the closed-loop eigenvalues in the interval with $a=-2$ and $b=-5$. By using the algorithm, the state feedback matrix obtained is: 


$$
K=\left[\begin{array}{cccccccccc}
-62.0827 & -35.4657 & 17.4115 & 4.2389 & -30.5613 & -53.0298 & 1.5823 & -28.9658 & 34.7812 & -2.9389 \\
24.5794 & 14.3848 & -6.7470 & 3.6703 & 9.5365 & 17.4210 & 3.1291 & 10.7556 & -8.2087 & 1.2975 \\
-54.7405 & -33.0166 & 17.3771 & 2.3426 & -28.8387 & -46.8042 & 0.3634 & -27.9290 & 28.0402 & -3.0393 \\
62.5201 & 34.4475 & -16.8606 & -0.4233 & 29.4655 & 51.0074 & -0.0145 & 26.0795 & -30.0149 & 3.2485 \\
4.5182 & 1.7631 & -2.9103 & -1.3963 & 0.9873 & 3.7016 & -0.5476 & 2.9036 & -3.5035 & -0.3685 \\
1.9439 & 1.0187 & 0.1789 & 1.0840 & 0.8108 & 1.8077 & -2.4683 & -2.0352 & -1.8540 & -0.3903
\end{array}\right]
$$

It can be verified that the closed-loop eigenvalues are $\{-2.5079 \pm 4.1980 i,-4.2020 \pm 1.6436 i,-3.1994 \pm 3.0331 i,-4.6899 \pm 0.6454 i,-2.0486,-3.9236\}$, clearly all are in the specified interval.

\section{Conclusion}

A simple algorithm was given for localization of eigenvalues in specified regions of complex plane by state feedback control. This method was achieved by implementing properties of vector companion forms. The merit of this approach is that it can be achieved by elementary similarity operations which are significantly simpler to realize computationally than the existing methods. This method can be used for large-scale continuous-time linear control systems as well. It is claimed that the transformations obtained by similarity operations reduce accuracy of the computations [3], however, other methods such as LQR methods [14] and gerschgorin [13] are more complicated.

\section{References}

[1] M.H. Amin, Optimal discrete systems with prescribed eigenvalues, International journal of control, 40 (1984), pp. 783-794.

[2] A. Arjmandzadeh, S. Effati and M. Zamirian, Interval Support Vector Machine in Regression Analysis, The Journal of Mathematics and Computer Science, 2 (2011),

pp. $565-571$

[3] P. Benner, M. Castillo and E.S. Quintana-orti, Partial stabilization of large-scale discrete-time linear control systems, Technical Report, University of Bremen, Germany, March 2001.

[4] J. H. Chou, Pole assignment robustness in a specified disk. Systems \& Control Letters, 16 (1991), pp. 41-44.

[5] M. M. Fateh, H. Ahsani Tehrani, S. M. Karbassi, Repetitive control of electrically driven robot manipulators, International Journal of Systems Science, Published Online: 18 Oct 2011.

[6] J. L. Figueroa and J. A. Romagnoli, An algorithm for robust pole assignment via polynomial approach. IEEE Transactions on Automatic Control, 39 (1994), pp. 831835.

[7] K. Furuta and S.B. Kim, Pole assignment in a specified disk. IEEE Transactions on Automatic Control, 32 (1987), pp. 423-427. 
[8] L. Grammont and A. Largillier, Krylov method revisited with an application to the localization of eigenvalues, Numerical Functional Analysis and Optimization, 27 (2006), pp. 583-618.

[9] A. H. Borzabadi and L. Heidarian, Determining the objective value range for a class of interval convex optimization problems, The Journal of Mathematics and Computer Science, 3 (2011), pp. 396 - 402

[10] S.M. Karbassi and D.J. Bell, Parametric time-optimal control of linear discrete-time systems by state feedback-Part 1: Regular Kronecker invariants, International journal of control, 57 (1993), pp. 817-830.

[11] S.M. Karbassi and D.J. Bell, Parametric time-optimal control of linear discrete-time systems by state feedback-Part 2: Irregular Kronecker invariants, International journal of control, 57 (1993), pp. 831-83.

[12] S.M. Karbassi and H.A. Tehrani, Parameterizations of the state feedback controllers for linear multivariable systems, Computers and Mathematics with Applications, 44 (2002), pp. 1057-1065.

[13] H.A. Tehrani, Assignment of Eigenvalues in a Disc D (c, r) of Complex Plane with Application of the Gerschgorin Theorem. World Applied Sciences Journal, 5 (5) (2008), pp. 576-581.

[14] L. Yuan, L.E.K. Achenie and W. Jiang, Linear quadratic optimal output feedback control for systems with poles in a specified region. International journal of control, 64 (1996), pp. 1151- 1164. 